\title{
Trastornos tiroideos por amiodarona
}

\section{The effects of amiodarone on the thyroid}

\author{
M. Toni ${ }^{1}$, E. Anda ${ }^{1}$, J. Pineda ${ }^{2}$, J.P. Martínez de Esteban ${ }^{1}$, M.D. Ollero ${ }^{1}$
}

\section{RESUMEN}

Fundamento. Muchos pacientes reciben tratamiento con amiodarona por su eficacia como antiarrítmico. Aproximadamente el $16 \%$ desarrollan alteraciones de la función tiroidea. El objetivo de este estudio es valorar la relevancia de dichas alteraciones en la población navarra, estudiada entre 2001 y 2007.

Material y métodos. Se trata de un estudio retrospectivo, en el que se han analizado las características de 182 pacientes remitidos a la consulta de endocrinología, por desarrollar disfunción tiroidea mientras se encontraban en tratamiento con amiodarona.

Para ello se han medido en plasma los niveles de hormonas tiroideas y anticuerpos antitiroideos y, cuando ha sido necesario, se ha realizado estudio tiroideo de imagen, ya sea mediante gammagrafía con tecnecio 99 o ecografía.

Resultados. El screening de la función tiroidea, recomendado previamente al inicio de tratamiento con amiodarona, se efectúo solamente en el $20,9 \%$ de los pacientes estudiados. El $41 \%$ presentaban hipotiroidismo; de éstos, en el $76 \%$ se suspendió el tratamiento con amiodarona. El tiempo medio de aparición del mismo fue de $21 \pm 12$ meses desde el inicio de la toma del fármaco. El 48,6\% desarrolló hipotiroidismo permanente. Este grupo presentaba niveles de TSH (tirotropina) mayores y llevaban menos tiempo de tratamiento con amiodarona.

El 59\% presentaban tirotoxicosis: el 59,4\% presentó una tirotoxicosis inducida por amiodarona (TIA) tipo 1, el $30,6 \%$ una TIA tipo 2 y el $10 \%$ restante fue etiquetada de tiroxicosis mixta. El tiempo medio de aparición de la tirotoxicosis fue de 29,5 meses desde el inicio del tratamiento. Los niveles de T4 libre eran significativamente mayores en la TIA tipo 2. Todos los pacientes fueron tratados con antitiroideos y/o corticoides, llegando a precisar en alguna ocasión ingreso hospitalario debido a la severidad del cuadro.

Conclusiones. En nuestra serie son más frecuentes las tirotoxicosis que el hipotiroidismo inducido por amiodarona ( $59 \%$ vs $41 \%$ ), lo que puede estar justificado por ser Navarra una comunidad con déficit de yodo. Es necesario el seguimiento de la función tiroidea durante al menos 2-3 años después del inicio del tratamiento con amiodarona.

Palabras clave. Amiodarona. Hipotiroidismo. Tirotoxicosis.

\begin{abstract}
Background. Amiodarone is a drug widely used for the treatment of arrhythmias. In 16\% of amiodarone-treated patients it causes changes in the thyroid function. The aim of this study was to determine the importance of amiodaroneinduced thyroid dysfunction in the population of Navarre studied between 2001 and 2007.

Methods. We present a retrospective study that considers the characteristics of 182 amiodarone-treated patients with thyroid dysfunction who had been referred to our Institute. We determined a series of biochemical and instrumental investigations (measurement of thyrotrophin, free thyroid hormones and thyroid autoantibodies; thyroid sonography and thyroid scintigraphy uptake).
\end{abstract}

Results. Screening of the thyroid function, recommended before starting amiodarone treatment, was carried out in only $20.9 \%$ of the patients. Forty-one percent of patients developed amiodarone induced hypothyroidism; in $76 \%$ of them the drug was withdrawn. Hypothyroidism appears after $21( \pm 12)$ months of amiodarone treatment. Forty-eight point six developed permanent hypothyroidism. This group of patients had higher serum levels of TSH (thyrotropin) and were treated for less time with amiodarone. Fifty-nine percent of patients developed amiodarone induced thyrotoxicosis; $59.4 \%$ were diagnosed with thyrotoxicosis (AIT) type $1,30.6 \%$ AIT type 2 and the remaining $10 \%$ were diagnosed with mixed thyrotoxicosis. Thyrotoxicosis appears after $29.5( \pm 17)$ months of amiodarone treatment.

The serum levels of free thyroxine were significantly higher in the AIT type 2 than in the AIT type1. All patients were treated with antithyroid drugs and/or corticoids. Some patients were admitted to hospital due to the severity of their illness.

Conclusions. In our study, amiodarone induced thyrotoxicosis was more frequent than hypothyroidism (59\% vs $41 \%$ ) because Navarre is an iodine-deficient area. It is necessary to control the thyroid function after 2-3 years of amiodarone treatment.

Key words. Amiodarone. Hypothyroidism. Thyrotoxicosis.

\section{An. Sist. Sanit. Navar. 2009; 32 (3): 363-370}

1. Servicio de Endocrinología. Hospital de Navarra. Pamplona

2. Servicio de Medicina Interna. Hospital García Orcoyen. Estella.

Recepción: 20 de abril de 2009

Aceptación provisional: 1 de junio de 2009

Aceptación definitiva: 1 de septiembre de 2009

\section{Correspondencia}

Marta Toni García

Servicio de Endocrinología

Hospital de Navarra

Irunlarrea, 3

31008 Pamplona

Tfno. 848422038/676890872 


\section{INTRODUCCIÓN}

Debido al frecuente uso de la amiodarona en nuestra comunidad, se ha detectado un importante porcentaje de alteraciones tiroideas secundarias a dicho tratamiento evaluadas en la consulta de endocrinología. El objetivo de este trabajo es revisar los pacientes estudiados entre 2001 y 2007 por este motivo y determinar el tipo de alteración tiroidea, así como el diagnóstico y tratamiento.

La amiodarona es un potente antiarrítmico de clase III, que posee una estructu- ra química parecida a la de las hormonas tiroideas (T3 y T4) ${ }^{1}$ (Fig. 1). Cada molécula de amiodarona contiene 2 átomos de yodo, lo que equivale al $37 \%$ de su peso molecular. Por lo que una dosis de mantenimiento habitual que suele ser de $200-600 \mathrm{mg} /$ día equivale a un aporte de $75-225 \mathrm{mg}$ de yodo orgánico. Tras su metabolismo se libera a la circulación un $10 \%$ de yodo en forma libre (7-22 mg), por lo que la dosis terapéutica de amiodarona supone un aporte de yodo de $50-100$ veces la ingesta diaria recomendada por la OMS, que es de 0,15-0,3mg/día ${ }^{2-3}$.

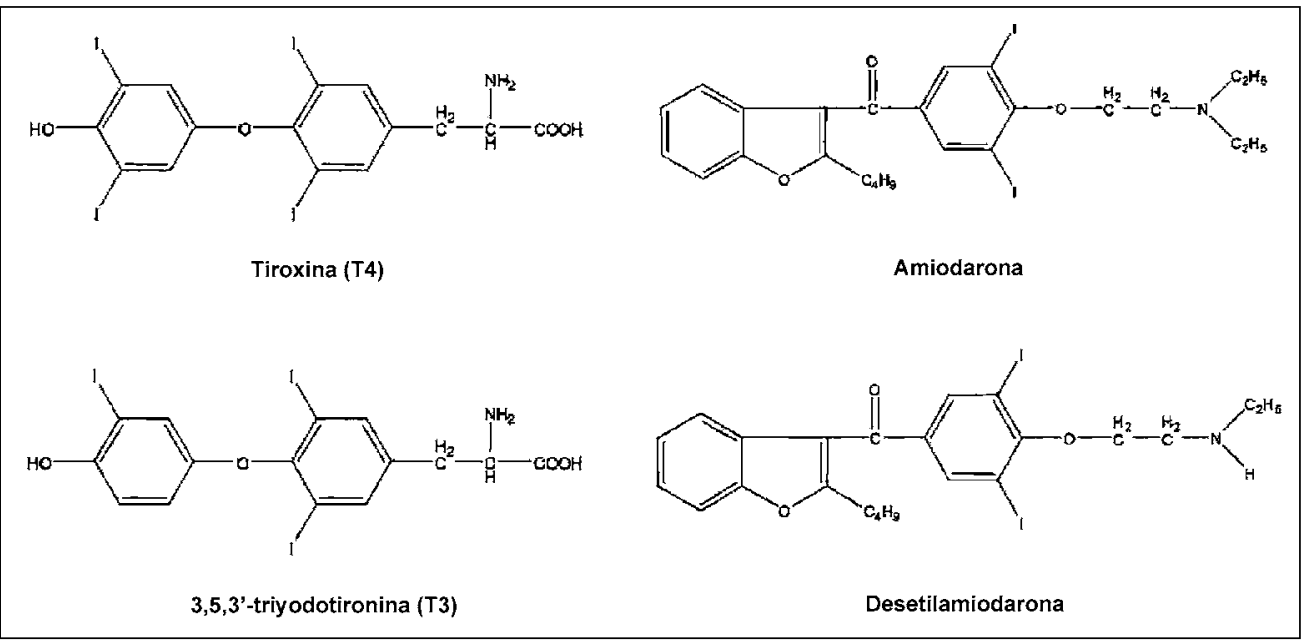

Figura 1. Estructura química de las hormonas tiroideas, amiodarona y su metabolito activo, desetilamiodarona².

La amiodarona es un fármaco muy utilizado para la prevención y el tratamiento de arritmias supraventriculares y ventriculares.

Aproximadamente el 15\% de los pacientes en tratamiento con amiodarona presentan efectos adversos en el primer año de tratamiento. Este porcentaje aumenta hasta un $50 \%$ si se emplea a más largo plazo ${ }^{4}$. Estos efectos secundarios pueden persistir meses después de la retirada del fármaco debido a su vida media prolongada (100 días) y a su capacidad para depositarse en el tejido adiposo. Se estima que en el $20 \%$ de los pacientes tratados crónicamente con amiodarona hay que retirarlo por efectos secundarios (Tabla 1$)^{5}$.
Tabla 1. Principales efectos adversos del tratamiento con amiodarona.

\begin{tabular}{lr}
\hline Microdepósitos corneales & $100 \%$ \\
Gastrointestinales (náuseas, anorexia) & $80 \%$ \\
Fotosensibilidad cutánea, decoloración & $55-75 \%$ \\
Ataxia, temblores, neuropatía & $48 \%$ \\
periférica & \\
Alteración función hepática & $25 \%$ \\
Disfunción tiroidea & $14-18 \%$ \\
Neumonitis intersticial, alteraciones & $10-13 \%$ \\
pulmonares & \\
Epididimitis & $11 \%$ \\
Bloqueo cardiaco, sinusal, bradicardia & $2-3 \%$ \\
Ginecomastia & excepcional \\
\hline
\end{tabular}


La disfunción tiroidea provocada por la amiodarona puede ser en forma tanto de tirotoxicosis (TIA) como de hipotiroidismo. Estas alteraciones pueden ser debidas a las propiedades intrínsecas del fármaco o a la sobrecarga de yodo que ocasiona ${ }^{6}$.

El hipotiroidismo inducido por amiodarona tiene una prevalencia variable, situada entre el 1 y el 25\%. Esta amplia variabilidad es debida a los distintos factores que influyen en su aparición como la ingesta habitual de yodo, el sexo femenino o la enfermedad tiroidea autoinmunitaria presente. Es más prevalente en áreas geográficas con aporte elevado de yodo ${ }^{7}$.

La TIA es más prevalente en las áreas con deficiencia de yodo ${ }^{8}$. Existen dos cuadros clínicos diferentes: TIA tipo 1 y TIA tipo 2. La TIA 1 se suele desarrollar en sujetos con enfermedad tiroidea de base y está causada por efecto Jod-Basedow (por sobrecarga de yodo), mientras que la TIA 2 ocurre en pacientes sin enfermedad tiroidea de base y es consecuencia de una tiroiditis destructiva inducida por el fármaco ${ }^{5}$. Así mismo, están descritas formas mixtas que comparten características de ambas y en las que las pruebas complementarias no son concluyentes ${ }^{9}$. El diagnóstico diferencial entre las distintas formas de tirotoxicosis es importante ya que el tratamiento es completamente distinto: la TIA tipol se trata fundamentalmente con antitiroideos y la tipo 2 con corticoides ${ }^{2,5,10}$.

Este diagnóstico diferencial se puede hacer fundamentalmente con gammagrafía tiroidea con tecnecio (alta captación en la tipo 1 y nula en la 2$)^{11}$, con ecografía doppler (vascularización aumentada en la tipo 1 y disminuida en la 2$)^{12}$ y recientemente, se ha comenzado a hablar del uso de la gammagrafía con sestaMIBI (cuya captación está aumentada en la tipo 1) 11,13.

\section{MATERIAL Y MÉTODOS}

Hemos analizado a todos los pacientes remitidos a la consulta de endocrinología entre 2001 y 2007, por presentar alteración de la función tiroidea secundaria a tratamiento con amiodarona. La remisión de estos pacientes se realizó tanto por el cardiólogo como por el médico de atención primaria. Se trata de una serie de 182 pacientes, 99 hombres y 83 mujeres con una edad media al diagnóstico de 68,9 $\pm 11,9$ años.

Las determinaciones hormonales y de anticuerpos antitiroideos se realizaron por quimiolumniniscencia (Immulite 2000), cuyos valores de referencia para TSH fueron 0,4 y $4 \mu \mathrm{U} / \mathrm{mL}$, para T4 libre 0,8 y $1,9 \mathrm{ng} / \mathrm{dL}$, para anticuerpos antitiroglobulina 0-20 U/ $\mathrm{mL}$ y para anticuerpos antiperoxidasa $0-10$ $\mathrm{U} / \mathrm{mL}$.

El $81,9 \%$ de los pacientes estaban en tratamiento con amiodarona por arritmia cardíaca tipo fibrilación auricular, el 9,3\% por taquicardia supraventricular y el $8,8 \%$ restante por otro tipo de arritmias cardiacas.

Estas alteraciones tiroideas secundarias al tratamiento con amiodarona representaron aproximadamente el $1 \%$ de las consultas nuevas realizadas en nuestro servicio.

Hemos realizado estudio estadístico utilizando el test de la t de Student, el coeficiente de correlación de Pearson y el test de Fisher cuando fue necesario. Se consideró como significativa una probabilidad menor de 0,05. Se utilizó el programa Statistical Package for Social Sciences (SPSS) versión 15.0 para Windows.

\section{RESULTADOS}

Si bien las guías tanto europeas como americanas recomiendan antes de iniciar el tratamiento con amiodarona, realizar una medición de las hormonas tiroideas para valorar una posible disfunción tiroidea, en nuestra serie de pacientes, solamente se llevó a cabo en el $20,9 \%$ de los pacientes (n:38).

En cuanto a las alteraciones tiroideas halladas, el 59\% (n:107) presentaban TIA y el 41\% (n:75) hipotiroidismo. Esta proporción puede estar basada en el hecho de que Navarra se considera una zona discretamente deficitaria en yodo, aunque podría 
estar sesgado con el hecho de que todas las tirotoxicosis son remitidas a nuestra consulta mientras que el hipotiroidismo puede ser seguido en el centro de salud sin precisar remisión al especialista.
En cuanto a las características basales de los pacientes, se trata de grupos homogéneos tanto en la edad, sexo, presencia de anticuerpos antitiroideos y duración del tratamiento con amiodarona (Fig. 2).

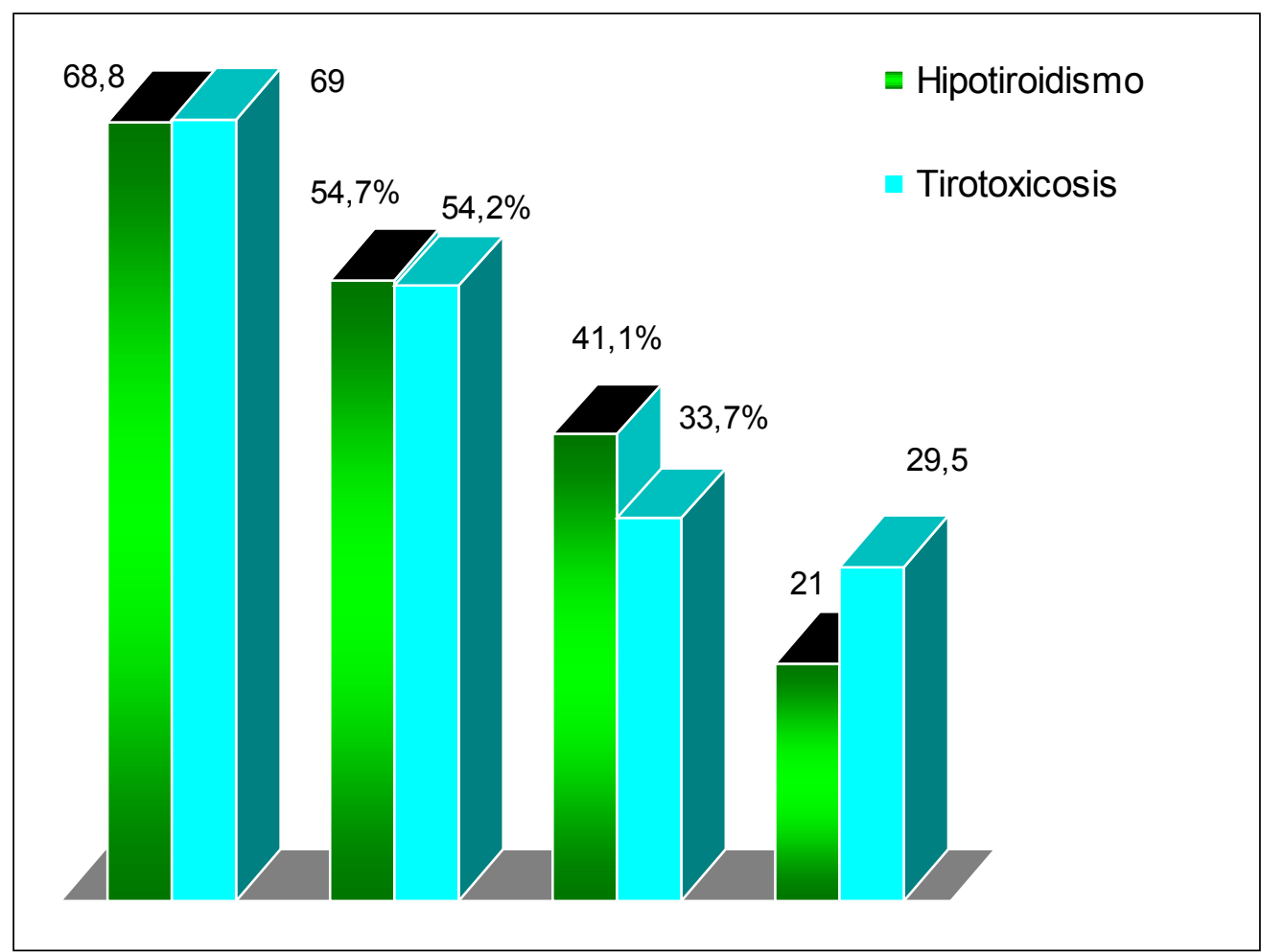

Figura 2. Características basales de la muestra.

\section{Hipotiroidismo}

Setenta y cinco pacientes presentaban hipotiroidismo estando en tratamiento con amiodarona. El 54,7\% eran hombres. El 84\% tenía una TSH superior a $10 \mathrm{mU} / \mathrm{l}$ y en el $44 \%$ de los casos se detectó un hipotiroidismo clínico $(\mathrm{T} 4 \mathrm{~L}<0,8)$.

El tiempo medio de seguimiento de los pacientes fue de 50,1 meses $( \pm 21,4$ meses $)$ : el 56\% desarrolló hipotiroidismo llevando más de año y medio de tratamiento con amiodarona. En el $76 \%$ de los pacientes se suspendió la amiodarona en el momen-
Edad (años) Sexo (varones) AC (+) Tto. amiodarona (meses) AC: anticuerpos, Tto: tratamiento to del diagnóstico de hipotiroidismo y así mismo se suplió la función tiroidea con levotiroxina.

El 51,4\% de los pacientes desarrollaron hipotiroidismo transitorio frente al 48,6\% que lo hizo de forma permanente. Las características de cada grupo se resumen en la tabla que se expone, encontrando diferencias significativas, en el tiempo de tratamiento con amiodarona, siendo mayor en el grupo que desarrolló el hipotiroidismo transitorio y en el nivel de TSH media, que fue mayor en el grupo que desarrolló el hipotiroidismo de forma permanente (Tabla 2). 
Tabla 2. Evolución de hipotiroidismo.

\begin{tabular}{lcc}
\hline & $\begin{array}{c}\text { Hipotiroidismo permanente } \\
\mathbf{n}: \mathbf{3 6}\end{array}$ & $\begin{array}{c}\text { Hipotirodismo transitorio } \\
\text { n: } \mathbf{3 9}\end{array}$ \\
\hline Sexo (hombres/mujeres) & $55,5 \% / 44,4 \%$ & $52,6 \% / 47,3 \%$ \\
Edad & 68,5 años $( \pm 10)$ & 69,1 años $( \pm 11,1)$ \\
AC & $36,1 \%$ & $44,7 \%$ \\
Tiempo tratamiento amiodarona & 18 meses $( \pm 10)$ & 30 meses $( \pm 17)^{*}$ \\
Mantiene amiodarona & $27,8 \%$ & $18,4 \%$ \\
TSH media & 39,5 mU/L $( \pm 17,4)$ & 19 mU/L $( \pm 6,9)^{*}$ \\
T4L $<0,8$ & $63,3 \%(\mathrm{n}: 21)$ & $36,4 \%(\mathrm{n}: 12)^{*}$ \\
\hline
\end{tabular}

*estadísticamente significativo $(\mathrm{p}<0,05)$

\section{Tirotoxicosis}

El 59,4\% desarrollaron TIA 1, frente al $30,6 \%$ que desarrollaron TIA 2. El 10\% de tirotoxicosis mixtas al diagnóstico inicial fueron posteriormente reasignadas como TIA 2 al estudiar la evolución de los pacien- tes (ausencia de patología tiroidea previa y posterior, y necesidad de corticoides en el seguimiento) ${ }^{8}$.

Las características basales de ambos grupos fueron homogéneas, siendo el nivel de T4 L la única diferencia significativa encontrada (Tabla 3 ).

Tabla 3. Características basales en los 2 tipos de tirotoxicosis.

\begin{tabular}{lrr}
\hline & \multicolumn{1}{c}{ TIA 1 } & \multicolumn{1}{c}{ TIA 2 } \\
\hline Edad & 69,6 años $( \pm 14,3)$ & 67,7 años $( \pm 11,8)$ \\
Sexo (hombres/mujeres) & $53,1 \% / 46,9 \%$ & $57,1 \% / 42,9 \%$ \\
Meses amiodarona & 29,4 meses $( \pm 34)$ & 22,2 meses $( \pm 21)$ \\
Anticuerpos positivos & $31,1 \%$ & $38,5 \%$ \\
T4L & $2,7 \mathrm{ng} / \mathrm{dL}( \pm 0,9)$ & $3,6 \mathrm{ng} / \mathrm{dL}( \pm 1,6)^{*}$ \\
TSH screening $<0,4$ & $2 / 23$ & $0 / 23$ \\
\hline
\end{tabular}

*estadísticamente significativo $(\mathrm{p}<0,05)$.

La dosis media de amiodarona que habían recibido los pacientes afectos de TIA 1 fue de $177,1 \mathrm{mg} /$ día $( \pm 31,3 \mathrm{mg} /$ día), y en aquellos con TIA 2 fue de $165,7 \mathrm{mg} /$ día $( \pm 27,2 \mathrm{mg} / \mathrm{d})$, no siendo estas diferencias estadísticamentes significativas $(p>0,05)$. El tiempo medio de seguimiento en estos pacientes fue de 43,5 meses $( \pm 28,9$ meses).

En lo referente a las características diagnósticas, sólo se realizó ecografía en el $20,5 \%$ de los pacientes, en la cual se hallaron nódulos tiroideos en el $76,5 \%$ de las TIA 1 y en ningún caso de las TIA
2. Nuestra principal herramienta para distinguir ambos tipos de tirotoxicosis además de la clínica, fue la gammagrafía tiroidea con Tc 99, que no mostró captación en el $100 \%$ de los pacientes diagnosticados de TIA 2.

El 7,1\% (n:3) de los pacientes diagnosticados de TIA 2 desarrollaron hipotiroidismo posterior (Tabla 4). No se ha encontrado significación estadística con la positividad de anticuerpos antitiroideos en aquellos pacientes que desarrollaron hipotiroidismo posterior, si bien la muestra es muy pequeña. 
Tabla 4. Características diagnósticas y evolución de la tirotoxicosis inducida por amiodarona tipo 1 y tipo 2.

\begin{tabular}{lrr}
\hline & TIA $\mathbf{1}$ & TIA 2 \\
\hline Bocio a la palpación & No: $54.7 \% ;$ & No: $47,6 \%$ \\
& Sí: $45,3 \%$ & Sí: $52,4 \%$ \\
\hline Eco realizada (sin utilizar doppler) & $26,6 \%$ (n: 17$)$ & $11,9 \%(\mathrm{n}: 5) *$ \\
\hline Nodular & $76,5 \%$ & $0 \% *$ \\
\hline Gammagrafía & captante: $33,3 \%$ & blanca: $100 \%(\mathrm{n}: 33) *$ \\
\hline Hipotiroidismo posterior & blanca: $66,7 \%(\mathrm{n}: 39)$ & $7,1 \%(\mathrm{n}: 3) *$ \\
\hline
\end{tabular}

*estadísticamente significativo $(\mathrm{p}<0,05)$

En cuanto al tratamiento, el 100\% de los pacientes con TIA 1 fueron tratados con antitiroideos frente al 83,3\% de las tipo 2 . La dosis media inicial fue mayor en este último grupo (18 $\mathrm{mg}$ frente a $15,5 \mathrm{mg}$ en el primero). Sin embargo, el tiempo medio de tratamiento farmacológico fue mayor en las TIA 1:8,2 meses frente 6,6 meses en las TIA 2. El tratamiento farmacológico se suspendió en el momento en que la función tiroidea fue controlada.

En todos los pacientes se suspendió el tratamiento con amiodarona, tal y como se recomienda en estos casos, siempre que sea posible desde el punto de vista cardiológico.

En ningún caso se registró recidiva de la TIA, ningún paciente de los evaluados precisó tratamiento con radioyodo, con cirugía ni con agentes colecistrográficos orales.

Se observaron efectos secundarios del tratamiento con antitiroideos en el 3\% (n:3): dos presentaron hepatotoxicidad y uno vasculitis.

Se registraron un total de 25 fallecimientos en nuestra serie, en 10 pacientes con hipotiroidismo y en 15 con tirotoxicosis (10 con TIA 1 y 5 con TIA 2 ). El tiempo medio desde el diagnóstico de la disfunción tiroidea hasta el exitus fue de 25,2 meses (1-69). Tenían una edad de 75,6 años en el momento del exitus y la causa del fallecimiento en el $48 \%$ fue la descompensación cardíaca.

\section{DISCUSIÓN}

La amiodarona es un antiarrítmico muy utilizado por su alta eficacia, pero tiene efectos secundarios frecuentes, siendo uno de ellos las alteraciones tiroideas. Por este motivo 182 pacientes han sido remitidos a nuestra consulta en un periodo de seis años.

En nuestra casuística, el porcentaje de tirotoxicosis inducida por amiodarona es mayor que el de hipotiroidismo, como corresponde al hecho de ser Navarra un área geográfica yodo-deficiente, coincidiendo con los datos publicados en otras zonas ${ }^{7}$.

En cuanto a la autoinmunidad, algunos autores han descrito que la amiodarona es capaz de inducir autoinmunidad tiroidea en más del $50 \%$ de los pacientes ${ }^{14}$. Si bien en nuestra serie no teníamos datos de autoinmunidad previa al tratamiento con amiodarona, el total de autoinmunidad positiva durante el mismo es de $35,2 \%$ lo que se corresponde con los resultados de otros autores ${ }^{15}$ que no han podido confirmar dicha teoría.

El hipotiroidismo en nuestra serie se produce en un porcentaje similar en hombres y mujeres. Este dato discrepa de lo encontrado en otros estudios en los que está descrito una mayor frecuencia en mujeres, con una relación mujer: varón de 1,5:1 y un riesgo relativo de $7,9^{16}$. Asimismo, el tiempo medio de aparición del hipotiroidismo en nuestra serie fue de $21 \pm 12$ 
meses desde el inicio de tratamiento con amiodarona, siendo mayor que el hallado en gran parte de la literatura. En la mayoría de los estudios se ha descrito la aparición del hipotiroidismo de manera temprana a la instauración del tratamiento, en torno a los 6-12 meses del inicio del mismo, siendo infrecuente después de los 18 meses $^{17}$, aunque en otras series también encuentran aparición de hipotiroidismo a más largo plazo, coincidiendo con nuestros datos ${ }^{18}$. Aunque no encontramos explicación para esta diferencia pensamos que puede tener una implicación clínica, al menos en nuestro medio y debamos continuar vigilando la función tiroidea a largo plazo.

En el subgrupo de pacientes que presentan un hipotiroidismo de forma permanente, el porcentaje de pacientes con positividad de anticuerpos antitiroideos es del $36,1 \%$. Habitualmente esta evolución del hipotiroidismo casi siempre está asociada a tiroiditis crónica autoinmune ${ }^{19}$, pero en nuestros pacientes no hemos encontrado diferencias significativas entre la presencia de anticuerpos antitiroideos en los pacientes con hipotiroidismo permanente $(36,1 \%)$ o transitorio (44,7\%).

Lo que sí nos puede orientar al desarrollo de hipotiroidismo permanente según los resultados obtenidos, son los mayores niveles de TSH. Así, en aquellos pacientes con TSH más elevada al diagnóstico, deberíamos mantener una actitud más conservadora a la hora de retirar el tratamiento sustitutivo con levotiroxina.

En lo referente a los pacientes que desarrollaron tirotoxicosis, en nuestra población es más prevalente la TIA 1, lo que también se correlaciona con el hecho de residir en un área con déficit de yodo, coincidente con los datos de la literatura ${ }^{18}$.

En nuestros pacientes afectos de TIA no hemos encontrado diferencias respecto al sexo. No obstante, en la literatura se ha descrito una mayor prevalencia en varones, con una relación varón:mujer de $3: 1^{20}$.

Tampoco hemos encontramos diferencias en la edad media de los pacientes que desarrollan hipotiroidismo y los que desa- rrollan tirotoxicosis, aunque en algunos estudios está descrito que éstos últimos suelen ser más jóvenes ${ }^{21}$. Los pacientes afectos de TIA fueron tratados con antitiroideos y/o corticoides, de manera similar a la de los grupos descritos en otras series ${ }^{5,8}$.

Para conseguir un correcto control fue preciso el ingreso hospitalario únicamente en 3 pacientes, de lo que podemos deducir que aunque la mayoría de pacientes pueden ser tratados de manera ambulatoria con buenos resultados, hay que tener en cuenta que existe un riesgo de mala evolución y es en estos casos, en los que debemos adoptar una postura terapéutica más agresiva, por el riesgo de mortalidad existente.

Este trabajo nos lleva a insistir principalmente en algunos aspectos:

- La necesidad de realización de screening de la función tiroidea previamente a iniciar el tratamiento con amiodarona.

- La continuidad del seguimiento de la función tiroidea durante al menos 2-3 años, ya que en el $56 \%$ de los casos el hipotiroidismo aparece tardíamente.

- La remisión de los pacientes con TIA a una consulta especializada dada la importancia del diagnóstico diferencial y de la potencial severidad del cuadro clínico.

\section{BIBLIOGRAFÍA}

1. Shezad Basaria, MD, David S. Cooper, MD. Amiodarone and the thyroid. Am J Med 2005; 118 : 706-714.

2. IgLESIAS P. Repercusiones del tratamiento con amiodarona sobre la función tiroidea y su manejo actual. Endocrinol Nutr 2007; 54: 354370 .

3. HaRJai KJ, Licata AA. Effects of amiodarone on thyroid function. Ann Intern Med 1997; 126 : 63-73.

4. Goldschlager N, Epstein AE, Naccarelli G, OlsHANSKY B, SINGH B. Practical guidelines for clinicians who treat patients with amiodarone. Practice Guidelines Subcommittee, North American society of pacing and electrophysiology. Arch Intern Med 2000; 160: 1741-1748. 
5. Enio M. Bartalena L, Bogazzi F, Braverman LE. The effects of amiodarone on the thyroid. Endocr Rev 2001; 22: 240-254.

6. Bogazzi F, Bartalena L, Gasperi M, Braverman LE, MARTINo E. The various effects of amiodarone on thyroid function. Thyroid 2001; 11: 511519.

7. Martino E, Safran M, Aghini-Lombardi F, RajataNAVIN R. LENZIARDI M, FAY M et al. Environmental iodine intake and thyroid dysfunction during chronic amiodarone therapy. Ann Intern Med 1984; 101: 28-34.

8. Basaria S, CoOper DS. Amiodarone and the thyroid. Am J Med 2005; 118: 706-714.

9. Franklyn JA, Gammage MD. Treatment of amiodarone-associted thyrotoxicosis. Nat Clin Pract Endocrinol Metab 2007; 3: 662-666.

10. Erdogan MF, Güleç S, Tutar E, Baskal N, ErdoGAN G. A stepwise approach to the treatment of amiodarone-induced thyrotoxicosis. Thyroid 2003; 13; 205-209.

11. Piga M, Cocco MC, Serra A, Boi F, Loy M, MaRIOTTI $\mathrm{S}$. The usefulness of $99 \mathrm{~m}$ - Tc-sestaMIBI thyroid scan in the differential diagnosis and management of amiodarone-induced thyrotoxicosis. Eur J Endocrinol 2008; 159: 423429.

12. Eaton SE, Euinton HA, Newman CM, WeEtman AP, BENNET WM. Clinical experience of amiodarone-induced thyrotoxicosis over a 3-year period: role of colour-flow Doppler sonography. Clin Endocrinol (Oxf) 2002; 56: 33-38.

13. Tanda ML, Bogazzi F, Martino E, Bartalena L. Amiodarone-induced thyrotoxicosis: something new to refine the inicial diagnosis? Eur J Endocrinol 2008; 159: 359-361.

14. Monteiro E, Galvao-Teles A, Santos ML, Mourao L, Correia MJ, Lopo Tuna J et al. Antithyroid antibodies as an early marker for thyroid disease induced by amiodarone. Br Med J 1986; 292: 227-228.

15. Safran M, Martino E, Aghini-Lombardi F, BartaleNA L, Balzano S, Pinchera A et al. Effect of amiodarone on circulating antithyroid antibodies. BMJ 1988; 297: 456-457.

16. TRIP MD, WiERSINGA W, Plomp TA. Incidence, predictability, and pathogenesis of amiodarone-induced thyrotoxicosis and hypothyroidism. Am J Med 1991; 91: 507-511.

17. Batcher EL, Tang XC, Singh BN, Singh SN, Reda DJ, HERShMAN JM. Thyroid function abnormalities during amiodarona therapy for persistent atrial fibrillation. Am J Med 2007; 120; 880-885.

18. Sato K, MiyaKawa M, Eto M, Inaba T, Matsuda N, SHIGA $\mathrm{T}$ el al. Clinical characteristics of amiodarone-induced thyrotoxicosis and hypothyroidism in Japan. Endocr J 1999; 46: 443-451.

19. Martino E, Aghini-Lombardi F, Mariotti S, Bartalena L, Lenziardi M, Ceccarelli C et al. Amiodarone iodine-induced hypothyroidism: risk factors and follow-up in 28 cases. Clin Endocrinol (Oxf) 1987; 26: 227-237.

20. Sato K, Miyakawa M, Eto M, Inaba T, Matsuda N, SHIGA T el al. Clinical characteristics of amiodarone-induced thyrotoxicosis and hypothyroidism in Japan. Endocr J 1999; 46: 443-451.

21. HARJAi KJ, LicATA AA. Amiodarone induced hyperthyroidism: a case series and brief review of literature. Pacing Clin Electrophysiol 1996; 19: 1548-1554.

22. Borowski GD, Garofano CD, Rose LI, Spielman SR, Rotmensch HR, Greenspan Am et al. Effect of long-term amiodarone therapy on thyroid hormone levels and thyroid function. Am J Med 1985; 78: 443-450. 